\title{
New computational method for solving fractional Riccati equation
}

\author{
Mohammed Ali*, Imad Jaradat, Marwan Alquran \\ Department of Mathematics, Jordan University of Science and Technology, Irbid 22110, Jordan.
}

\begin{abstract}
In this work, we implement the residual power series (RPS) method for solving the time fractional nonlinear Riccati initial value problem

$$
\left\{\begin{array}{l}
D_{t}^{\alpha} y(t)+a y(t)+b y^{2}(t)=c, \quad 0<\alpha \leqslant 1,0 \leqslant t<R, \\
y(0)=d,
\end{array}\right.
$$

where $a, b, c, d$ are constants and $D_{t}^{\alpha}$ is the Caputo fractional derivative. An analytical solution of $y(t)$ is obtained as a convergent fractional power series in $t$. To demonstrate the dependability of the proposed method, three illustrative examples are offered and the obtained results are compared with some existing results in the literature. Moreover, the results show that the approximate solutions are continuously communicate, as $\alpha$ increases, until the first derivative is reached. (c)2017 all rights reserved.
\end{abstract}

Keywords: Fractional Riccati, Caputo derivative, residual power series.

2010 MSC: 26A33, 35F25, 35C10.

\section{Introduction}

Nonlinear fractional equations play a major role in various fields, such as fluid mechanics, optical fibers, solid state physics, geochemistry, and so on. This is due to the fact that fractional derivatives can grasp the history of the variable under consideration. Exact solutions for the majority of fractional differential equations (FDEs) cannot be found easily, thus analytical and numerical methods must be used. In fact, several numerical methods for solving FDEs have been presented in the literature and they have their own advantages and limitations.

In the present work, we mainly deal with a new analytical method proposed by Arqub in 2013 to solve fuzzy differential equations [1]. Later, this method was modified [9] and applied successfully to several kinds of classical and fractional differential equations $[2-5,10,11,13,16,17,23,24]$. The main feature of RPS method is that during the transition from simple linearity to complex nonlinearity, there is no need for any conversion. This makes the RPS method suitable for solving nonlinear problems [16].

The motivation of this paper is to apply the residual power series (RPS) method to solve the quadratic Riccati differential equation of fractional order which reads

\footnotetext{
${ }^{*}$ Corresponding author

Email addresses: myali@just.edu.jo (Mohammed Ali), iajaradat@just.edu.jo (Imad Jaradat), marwan04@just.edu.jo (Marwan Alquran)
}

doi:10.22436/jmcs.017.01.10 


$$
D_{t}^{\alpha} y(t)+a y(t)+b y^{2}(t)=c,
$$

with initial condition $y(0)=d$, where $\alpha$ is the fractional derivative in the Caputo sense and $0<\alpha \leqslant 1$. The Riccati differential equation is named after the Italian Nobleman Count Jacopo Francesco Riccati $(1676-1754)$. Due to the important role for these equations in optimal control and diffusion problems [6], many authors have made attempts to solve these equations using different numerical methods. Such methods are: the Laplace-Adomian-Pade method [15], the modified homotopy perturbation method [20], the He's variational iteration method [14], the Chebyshev wavelet operational matrix method [18], the finite difference method and Pade-variational iteration method [22] and the shifted Jacobi polynomial integral operational matrix method [19].

The paper is organized as follows: in Section 2, some definitions and theorems regarded Caputo's derivative and fractional power series are given. Detailed derivation of the RPS solution of the fractional Riccati has been discussed in Section 3. Finally, the performance of the RPS method has been tested in Section 4.

\section{Preliminaries}

Many definitions and studies of fractional calculus have been proposed in the literature. These definitions include: Grunwald-Letnikov, Riemann-Liouville, Weyl, Riesz and Caputo sense. In the Caputo case, the derivative of a constant is zero and one can define, properly, the initial conditions for the fractional differential equations which can be handled by using an analogy with the classical integer case. For these reasons, researchers prefer to use the Caputo fractional derivative $[7,8,12,21]$ which is defined as follows.

Definition 2.1. Let $m$ be the smallest integer that exceeds $\alpha$, the Caputo fractional derivatives of order $\alpha>0$ is defined as

$$
D^{\alpha} y(t)=\frac{d^{\alpha} y(t)}{d t^{\alpha}}= \begin{cases}\frac{1}{\Gamma(m-\alpha)} \int_{0}^{t}(t-\tau)^{m-\alpha-1} \frac{d^{m} y(\tau)}{d \tau^{m}} d \tau & : m-1<\alpha<m, \\ \frac{d^{m} y(t)}{d t^{m}} & : \alpha=m \in \mathbb{N} .\end{cases}
$$

As a direct implementation, the Caputo fractional derivative of the power function satisfies

$$
\mathrm{D}^{\alpha} \mathrm{t}^{\mathrm{p}}=\left\{\begin{array}{cc}
\frac{\Gamma(p+1)}{\Gamma(p-\alpha+1)} \mathrm{t}^{p-\alpha}: & \mathrm{m}-1<\alpha<\mathrm{m}, \quad \mathrm{p}>\mathrm{m}-1, \\
0 & : \mathrm{m}-1<\alpha<\mathrm{m}, \quad \mathrm{p} \leqslant \mathrm{m}-1 .
\end{array}\right.
$$

Now, we survey some needed definitions and theorems regarded the fractional power series (FPS) also, more information can be found in $[1,2,9]$.

Definition 2.2. A power series expansion of the form

$$
\sum_{m=0}^{\infty} c_{m}\left(t-t_{0}\right)^{m \alpha}=c_{0}+c_{1}\left(t-t_{0}\right)^{\alpha}+c_{2}\left(t-t_{0}\right)^{2 \alpha}+\cdots,
$$

where $0 \leqslant m-1<\alpha \leqslant m, t \leqslant t_{0}$ is called fractional power series PS about $t=t_{0}$.

Theorem 2.3. Suppose that $\mathrm{f}$ has a fractional PS representation at $\mathrm{t}=\mathrm{t}_{0}$ of the form

$$
f(t)=\sum_{m=0}^{\infty} c_{m}\left(t-t_{0}\right)^{m \alpha}, \quad 0 \leqslant m-1<\alpha \leqslant m, t_{0} \leqslant t<t_{0}+R .
$$

If $\mathrm{D}^{\mathrm{m} \alpha} \mathrm{f}(\mathrm{t})$ are continuous on $\left(\mathrm{t}_{0}, \mathrm{t}_{0}+\mathrm{R}\right)$ for $\mathrm{m} \in \mathbb{N}$, then $\mathrm{c}_{\mathrm{m}}=\frac{\mathrm{D}^{\mathrm{m} \alpha} \mathrm{f}\left(\mathrm{t}_{0}\right)}{\Gamma(\mathrm{m} \alpha+1)}$ and $\mathrm{R}$ is the radius of convergence.

Next, we present in details the derivation of the residual power series solution to the fractional Riccati equation. 


\section{Residual power series (RPS) for solving fractional Riccati equation}

Consider the fractional Riccati initial value problem

$$
\left\{\begin{array}{l}
D_{t}^{\alpha} y(t)+a y(t)+b y^{2}(t)=c, \\
y(0)=d,
\end{array}\right.
$$

where $0<\alpha \leqslant 1,0 \leqslant t<R$, and $a, b, c, d \in \mathbb{R}$. The RPS method propose the solution for Eq. (3.1) as a fractional power series (FPS) about the initial point $t=0$ of the form

$$
y(t)=\sum_{n=0}^{\infty} \frac{a_{n}}{\Gamma(n \alpha+1)} t^{n \alpha}, \quad 0<\alpha \leqslant 1,0 \leqslant t<R .
$$

Apparently, according to the initial condition, it yields that $a_{0}=d$. Next, we let $y_{k}(t)$ to denote the $k$-th truncated series of $y(t)$ for $k \geqslant 1$, which can be used to approximate the solution, i.e.,

$$
y_{k}(t)=d+\sum_{n=1}^{k} \frac{a_{n}}{\Gamma(n \alpha+1)} t^{n \alpha}, \quad 0<\alpha \leqslant 1,0 \leqslant t<R .
$$

Now, we define the residual function, $\operatorname{Res}_{y}(t)$, for Eq. (3.1) as

$$
\operatorname{Res}_{y}(t)=D_{t}^{\alpha} y(t)+a y(t)+b y^{2}(t)-c
$$

and accordingly, the $k$-th residual function, $\operatorname{Res}_{y, k}(t)$, is

$$
\operatorname{Res}_{y, k}(t)=D_{t}^{\alpha} y_{k}(t)+a y_{k}(t)+b y_{k}^{2}(t)-c .
$$

It is clear that $\lim _{k \rightarrow \infty} \operatorname{Res}_{y, k}(t)=\operatorname{Res}_{y}(t)=0$ for all $t \geqslant 0$. As the fractional derivative of a constant function in the Caputo's sense is 0 , we have $D_{t}^{n \alpha} \operatorname{Res}_{y}(t)=0$. Also, the fractional derivative $D_{t}^{n \alpha}$ of $\operatorname{Res}_{y}(t)$ and $\operatorname{Res}_{y, k}(t)$ are matching at $t=0$ for each $n=0,1,2, \ldots, k$.

To obtain the value of the coefficients $a_{i}, i=1,2,3, \ldots, k$ in Eq. (3.2), we substitute the $k$-th truncated series of $y(t)$ into Eq. (3.3) and by using the fact $[1,9]$ that

$$
\mathrm{D}_{\mathrm{t}}^{(\mathrm{k}-1) \alpha} \operatorname{Res}_{\mathrm{y}, \mathrm{k}}(0)=0,0<\alpha \leqslant 1, k=1,2,3 \ldots,
$$

we obtain an algebraic system in $a_{i}, i=1,2,3, \ldots, k$.

Now, we explicitly apply the previous discussion to find $a_{i}$ under our consideration.

First, to determine $a_{1}$, we consider $(k=1)$ in (3.3)

$$
\operatorname{Res}_{y, 1}(t)=D_{t}^{\alpha} y_{1}(t)+a y_{1}(t)+b y_{1}^{2}(t)-c .
$$

But, $y_{1}(t)=d+\frac{a_{1}}{\Gamma(\alpha+1)} t^{\alpha}$. Therefore,

$$
\operatorname{Res}_{y, 1}(t)=a_{1}+a\left(a_{0}+\frac{a_{1}}{\Gamma(\alpha+1)} t^{\alpha}\right)+b\left(a_{0}+\frac{a_{1}}{\Gamma(\alpha+1)} t^{\alpha}\right)^{2}-c .
$$

From Eq. (3.4) we deduce that $\operatorname{Res}_{y, 1}(0)=0$. Thus,

$$
a_{1}=-a_{0} a-b a_{0}^{2}+c,
$$

where $a_{0}=d$.

Second, to obtain $a_{2}$, we substitute the 2-nd truncated series $y_{2}(t)=a_{0}+\frac{a_{1}}{\Gamma(1+\alpha)} t^{\alpha}+\frac{a_{2}}{\Gamma(1+2 \alpha)} t^{2 \alpha}$ into the 2-nd residual function $\operatorname{Res}_{y, 2}(t)$, i.e., 


$$
\begin{aligned}
\operatorname{Res}_{y, 2}(t)= & a_{1}+\frac{a_{2}}{\Gamma(\alpha+1)} t^{\alpha}+a\left(a_{0}+\frac{a_{1}}{\Gamma(\alpha+1)} t^{\alpha}+\frac{a_{2}}{\Gamma(2 \alpha+1)} t^{2 \alpha}\right) \\
& +b\left(a_{0}+\frac{a_{1}}{\Gamma(\alpha+1)} t^{\alpha}+\frac{a_{2}}{\Gamma(2 \alpha+1)} t^{2 \alpha}\right)^{2}-c .
\end{aligned}
$$

Applying $D_{t}^{\alpha}$ on both sides of Eq. (3.5) and solving $D_{t}^{\alpha} \operatorname{Res}_{y, 2}(0)=0$, will produce the same result as if we just consider the coefficient of the variable $t^{\alpha}$ in the expansion of (3.5) and multiply it by $\Gamma(\alpha+1)$. This argument is based on the fact that by Caputo derivative, $D_{t}^{\alpha}\left(t^{\alpha}\right)=\Gamma(\alpha+1)$ and $\left.D_{t}^{\alpha}\left(t^{b}\right)\right|_{t=0}=0, b>\alpha$. Thus, we get

$$
a_{2}=-\left(a+2 b a_{0}\right) a_{1}
$$

Finally, we can obtain the other coefficients $a_{k}, k \geqslant 3$ by considering the $k$-th residual function $\operatorname{Res}_{y, k}(t)$ and finding the coefficient of the variable $t^{(k-1) \alpha}$. Then, multiplying the obtained coefficient by the factor $\Gamma((k-1) \alpha+1)$ and by running this process, we have the following recurrence relation for $k \geqslant 2$

$$
a_{k+1}=\sum_{\substack{i+j=k \\ i, j \in \mathbb{Z}^{+}}} \frac{k \Gamma(k \alpha+1)}{\Gamma(i \alpha+1) \Gamma(j \alpha+1)} a_{i} a_{j} b-\left(a+2 a_{0} b\right) a_{k},
$$

where

$$
\kappa= \begin{cases}-2 & : i+j \neq k, \\ -1 & : i+j=k .\end{cases}
$$

It should be noted here that the RPS method is a numerical technique based on the generalized Taylor series formula which constructs an analytical solution in the form of a convergent series. Therefore, one can achieve a good approximation with the exact solution by using few terms only and thus, the overall errors can be made smaller by adding more new terms of the RPS approximations.

\section{Solutions and effects of fractional order of Riccati's}

The aim of this section is to study the solutions and the effects of the fractional order derivative to Riccati's equations. Based on the existence of a closed-form solution and on other applied numerical methods to Riccati's equations, three examples are considered to verify the applicability of the proposed algorithm.

Example 4.1. We first consider the following fractional Riccati equation, which is usually encountered in optimal control problems,

$$
D_{t}^{\alpha} y(t)-2 y(t)+y^{2}(t)=1,0<\alpha \leqslant 1, t \geqslant 0,
$$

subject to the homogeneous initial condition $y(0)=0$. Here, $a=-2, b=1, c=1$, and $a_{0}=d=0$. In the light of RPS algorithm discussed in Section 3, the proposed fractional power series solution for equation (4.1) is of the form

$$
y(t)=\frac{a_{1}}{\Gamma(\alpha+1)} t^{\alpha}+\frac{a_{2}}{\Gamma(2 \alpha+1)} t^{2 \alpha}+\frac{a_{3}}{\Gamma(3 \alpha+1)} t^{3 \alpha}+\frac{a_{4}}{\Gamma(4 \alpha+1)} t^{4 \alpha}+\cdots
$$

Following the recurrence relation (3.6) of $a_{n}$, the first few coefficients $a_{n}$ are

$$
\begin{aligned}
& a_{1}=1, \\
& a_{2}=2,
\end{aligned}
$$




$$
\begin{aligned}
& a_{3}=4-\frac{\Gamma(2 \alpha+1)}{\Gamma^{2}(\alpha+1)} \\
& a_{4}=2 a_{3}-\frac{4 \Gamma(3 \alpha+1)}{\Gamma(2 \alpha+1) \Gamma(\alpha+1)} \\
& a_{5}=2 a_{4}-\frac{2 \Gamma(4 \alpha+1)}{\Gamma(3 \alpha+1) \Gamma(\alpha+1)} a_{3}-\frac{4 \Gamma(4 \alpha+1)}{\Gamma^{2}(2 \alpha+1)} \\
& a_{6}=2 a_{5}-\frac{2 \Gamma(5 \alpha+1)}{\Gamma(4 \alpha+1) \Gamma(\alpha+1)} a_{4}-\frac{4 \Gamma(5 \alpha+1)}{\Gamma(3 \alpha+1) \Gamma(2 \alpha+1)} a_{3}, \\
& a_{7}=2 a_{6}-\frac{2 \Gamma(6 \alpha+1)}{\Gamma(5 \alpha+1) \Gamma(\alpha+1)} a_{5}-\frac{4 \Gamma(6 \alpha+1)}{\Gamma(4 \alpha+1) \Gamma(2 \alpha+1)} a_{4}-\frac{\Gamma(6 \alpha+1)}{\Gamma^{2}(3 \alpha+1)} a_{3}^{2}, \\
& a_{8}=2 a_{7}-\frac{2 \Gamma(7 \alpha+1)}{\Gamma(6 \alpha+1) \Gamma(\alpha+1)} a_{6}-\frac{4 \Gamma(7 \alpha+1)}{\Gamma(5 \alpha+1) \Gamma(2 \alpha+1)} a_{5}-\frac{2 \Gamma(7 \alpha+1)}{\Gamma(4 \alpha+1) \Gamma(3 \alpha+1)} a_{3} a_{4},
\end{aligned}
$$

and so on. In particular when $\alpha=1$, we have $a_{1}=1, a_{2}=1, a_{3}=2, a_{4}=-8, a_{5}=-56, a_{6}=-112$, $a_{7}=848, a_{8}=9088$. Therefore, the RPS solution to equation (4.1) of the first order is

$$
y(t)=t+t^{2}+\frac{t^{3}}{3}-\frac{t^{4}}{3}-\frac{7 t^{5}}{15}-\frac{7 t^{6}}{45}+\frac{53 t^{7}}{315}+\frac{71 t^{8}}{315}+\mathcal{O}\left(t^{9}\right)
$$

which is identical with the terms of the Taylor series expansion for the exact solution

$$
y(t)=1+\sqrt{2} \tanh \left(\sqrt{2} t+\frac{1}{2} \log \left(\frac{\sqrt{2}-1}{\sqrt{2}+1}\right)\right) .
$$

Figure 1 shows the effect of different values of the fractional derivative order $0<\alpha \leqslant 1$ for the approximate solutions $y_{10}(t), t \in I=[0,0.5]$. It is clear that the approximate solution $y_{10}(t)$ when $\alpha=1$ from our algorithm is in high agreement with the exact solution on I. Moreover, the approximate solutions $y_{10}(t)$ for different values of $0<\alpha \leqslant 1$ continuously approaches, as $\alpha$ increases, to the exact solution when $\alpha=1$. Thus, we anticipate a veracious solution for various values of $\alpha$.

In order to do further analysis, Table 1 shows a comparison between our approach and other existing numerical methods for $\alpha=0.5$ and $\alpha=0.75$. It should be noted that we compare only the 10-term approximate solution we obtained by RPS with third-component approximate solution (16-term) obtained by Laplace-Adomian-Pade method (LAPM) [15], the fourth-component approximate solution (16-term) obtained by Modified Homotopy Perturbation method (HPM) [20], and with 192-block pulse functions obtained by Chebyshev Wavelets (CW) [18]. It can be deduced from the table that the results obtained by the 10-term RPS approximate solution compare very well with other methods, especially when $\alpha$ gets close to 1 , but with easily computable components. Thus, more accuracy can be further achieved for various values of $\alpha$ by adding more terms.

Table 1: Comparison for the solution of Example 4.1 with $\alpha=0.5, \alpha=0.75$.

\begin{tabular}{ccccccccr}
\hline \hline & \multicolumn{3}{c}{$\alpha=0.5$} & & & $\alpha=0.75$ \\
$\mathrm{t}$ & RPS & HPM & CW & LAPM & RPS & HPM & CW & LAPM \\
\hline \hline 0.1 & 0.593178 & 0.321730 & 0.592756 & 0.356803 & 0.245431 & 0.216866 & 0.310732 & 0.193401 \\
0.2 & 0.955952 & 0.629666 & 0.933179 & 0.922865 & 0.475107 & 0.428892 & 0.584307 & 0.454602 \\
0.3 & 1.389321 & 0.940941 & 1.173983 & 1.634139 & 0.710342 & 0.654614 & 0.822173 & 0.784032 \\
0.4 & 2.351819 & 1.250737 & 1.346654 & 2.204441 & 0.941954 & 0.891404 & 1.024974 & 1.161985 \\
0.5 & 4.693042 & 1.549439 & 1.473887 & 2.400447 & 1.169808 & 1.132763 & 1.198621 & 1.543881 \\
\hline \hline
\end{tabular}




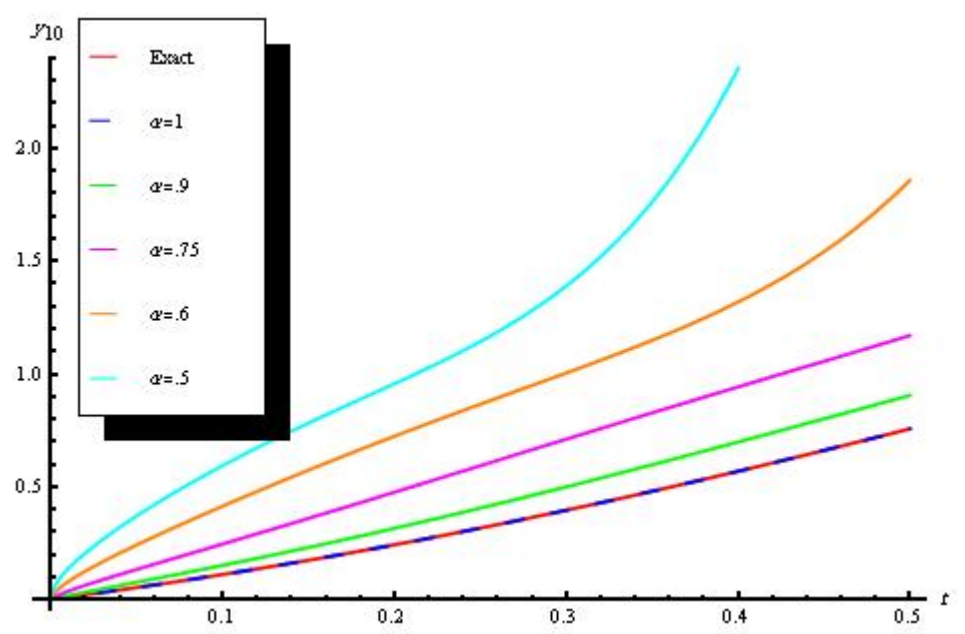

Figure 1

Example 4.2. Next, we consider the fractional Riccati equation

$$
D_{t}^{\alpha} y(t)+y^{2}(t)=1,0<\alpha \leqslant 1, t \geqslant 0,
$$

subject to the homogeneous initial condition $y(0)=0$. By running the recurrence relation (3.6) of $a_{n}$ with $a=0, b=1, c=1, a_{0}=d=0$, we get $a_{n}=0$ if $n$ is positive even integer,

$$
\begin{aligned}
& a_{1}=1, \\
& a_{3}=-\frac{\Gamma(2 \alpha+1)}{\Gamma^{2}(\alpha+1)}, \\
& a_{5}=\frac{2 \Gamma(4 \alpha+1) \Gamma(2 \alpha+1)}{\Gamma(3 \alpha+1) \Gamma^{3}(\alpha+1)}, \\
& a_{7}=-\frac{\Gamma(6 \alpha+1) \Gamma(2 \alpha+1)(4 \Gamma(4 \alpha+1) \Gamma(3 \alpha+1)+\Gamma(5 \alpha+1) \Gamma(2 \alpha+1))}{\Gamma(5 \alpha+1) \Gamma^{2}(3 \alpha+1) \Gamma^{4}(\alpha+1)},
\end{aligned}
$$

and so on. Therefore, the solution of (4.3) in the sense of RPS-algorithm has the form

$$
y(t)=\frac{a_{1}}{\Gamma(\alpha+1)} t^{\alpha}+\frac{a_{3}}{\Gamma(3 \alpha+1)} t^{3 \alpha}+\frac{a_{5}}{\Gamma(5 \alpha+1)} t^{5 \alpha}+\frac{a_{7}}{\Gamma(7 \alpha+1)} t^{7 \alpha}+\cdots
$$

In particular when $\alpha=1$, we have $y(t)=t-\frac{t^{3}}{3}+\frac{2 t^{5}}{15}-\frac{17 t^{7}}{315}+\mathcal{O}\left(t^{9}\right)$ which coincides precisely with the Taylor series expansion for the exact solution $y(t)=\left(e^{2 t}-1\right)\left(e^{2 t}+1\right)^{-1}$.

Figure 2 shows the approximate solutions $y_{10}(t)$ for $t \in I=[0,0.5]$ obtained by the RPS-algorithm for various values of $0<\alpha \leqslant 1$. Apparently, $y_{10}(t)$ for $\alpha=1$ is in high agreement with the exact solution on I. Moreover, $y_{10}(t)$ for different values of $\alpha$ continuously communicates until $\alpha=1$ is reached. Thus, a convenient solution is expected for various values of $\alpha$.

In Table 2, our numerical results are compared with other existing two methods for $\alpha=0.5$ and $\alpha=0.75$. Here we only choose the 10-term approximation obtained by the RPS-algorithm to be compared with the fourth-component approximate solution (16-term) obtained by Modified Homotopy Perturbation method (HPM) [20] and with 12-component approximate solution (12-term) obtained by LaplaceAdomian-Pade method (LAPM) [15]. It can be seen that our results are in accord with their results but with less computations. 


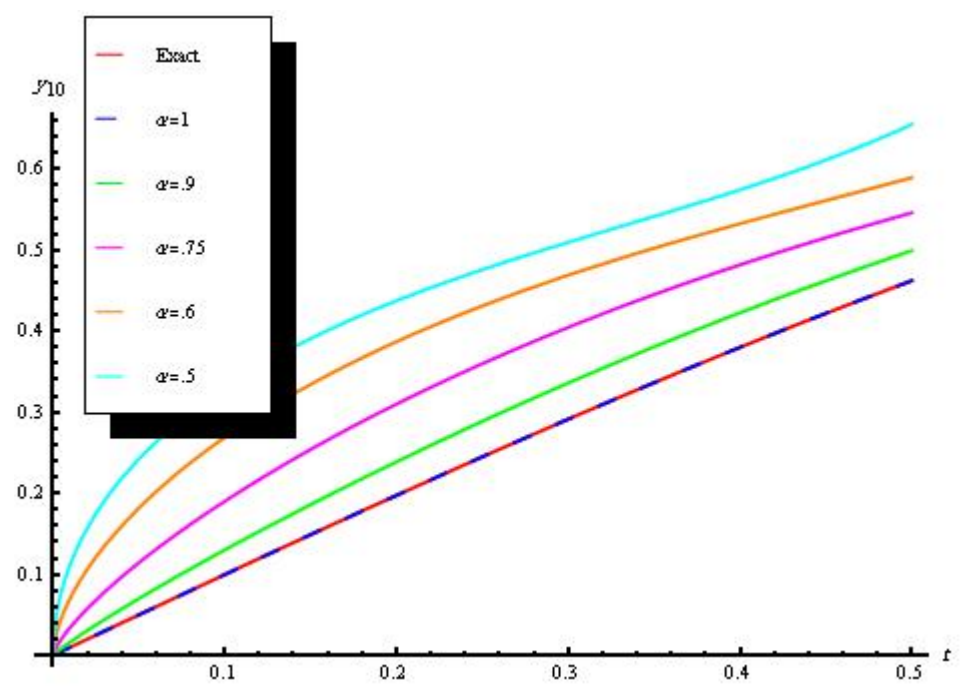

Figure 2

Table 2: Comparison for the solution of Example 4.2 with $\alpha=0.5, \alpha=0.75$.

\begin{tabular}{ccccccc}
\hline \hline \multicolumn{3}{c}{$\alpha=0.5$} & & \multicolumn{4}{c}{$\alpha=0.75$} \\
$\mathrm{t}$ & RPS & HPM & LAPM & RPS & HPM & LAPM \\
\hline \hline 0.1 & 0.33012277 & 0.273875 & 0.698739 & 0.19010097 & 0.184795 & 0.736836 \\
0.2 & 0.43740644 & 0.454125 & 0.785655 & 0.30997577 & 0.313795 & 0.870182 \\
0.3 & 0.50958082 & 0.573932 & 0.825857 & 0.40462777 & 0.414562 & 0.914950 \\
0.4 & 0.57432474 & 0.644422 & 0.850066 & 0.48176169 & 0.492889 & 0.935903 \\
0.5 & 0.65491555 & 0.674137 & 0.866733 & 0.54585356 & 0.462117 & 0.947342 \\
\hline \hline
\end{tabular}

Example 4.3. Lastly, we consider the fractional Riccati equation

$$
D_{t}^{\alpha} y(t)+y(t)-y^{2}(t)=0,0<\alpha \leqslant 1, t \geqslant 0,
$$

with nonhomogeneous initial condition $y(0)=\frac{1}{2}$. By applying the recurrence relation (3.6) of $a_{n}$ with $a=1, b=-1, c=0$, and $a_{0}=d=\frac{1}{2}$, we have $a_{n}=0$ if $n$ is even integer,

$$
\begin{aligned}
& \mathrm{a}_{1}=-\frac{1}{4}, \\
& \mathrm{a}_{3}=\frac{\Gamma(2 \alpha+1)}{16 \Gamma^{2}(\alpha+1)}, \\
& \mathrm{a}_{5}=-\frac{\Gamma(4 \alpha+1) \Gamma(2 \alpha+1)}{32 \Gamma(3 \alpha+1) \Gamma^{3}(\alpha+1)}, \\
& \mathrm{a}_{7}=\frac{\Gamma(6 \alpha+1) \Gamma(2 \alpha+1)(4 \Gamma(4 \alpha+1) \Gamma(3 \alpha+1)+\Gamma(5 \alpha+1) \Gamma(2 \alpha+1))}{256 \Gamma(5 \alpha+1) \Gamma^{2}(3 \alpha+1) \Gamma^{4}(\alpha+1)},
\end{aligned}
$$

and so on. Thereby, the solution of (4.4) in the sense of RPS-algorithm has the form

$$
y(t)=\frac{1}{2}+\frac{a_{1}}{\Gamma(\alpha+1)} t^{\alpha}+\frac{a_{3}}{\Gamma(3 \alpha+1)} t^{3 \alpha}+\frac{a_{5}}{\Gamma(5 \alpha+1)} t^{5 \alpha}+\frac{a_{7}}{\Gamma(7 \alpha+1)} t^{7 \alpha}+\cdots .
$$


Particularly with $\alpha=1$, we have $y(t)=\frac{1}{2}-\frac{t}{4}+\frac{t^{3}}{48}-\frac{t^{5}}{480}+\frac{17 t^{7}}{80640}+\mathcal{O}\left(t^{9}\right)$ which coincides precisely with the Taylor series expansion of the exact solution $y(t)=e^{-t}\left(1+e^{-t}\right)^{-1}$.

Figure 3 shows the behavior of the approximate solutions $y_{10}(t), t \in I=[0,0.5]$ for various values of $0<\alpha \leqslant 1$. Clearly, the approximate solution $y_{10}(t)$ when $\alpha=1$ from the RPS-algorithm is in harmony with the exact solution on I. Moreover, $y_{10}(t)$ for different values of $\alpha$ continuously communicates until $\alpha=1$ is reached. Thus, a convenient solution is expected for various values of $\alpha$.

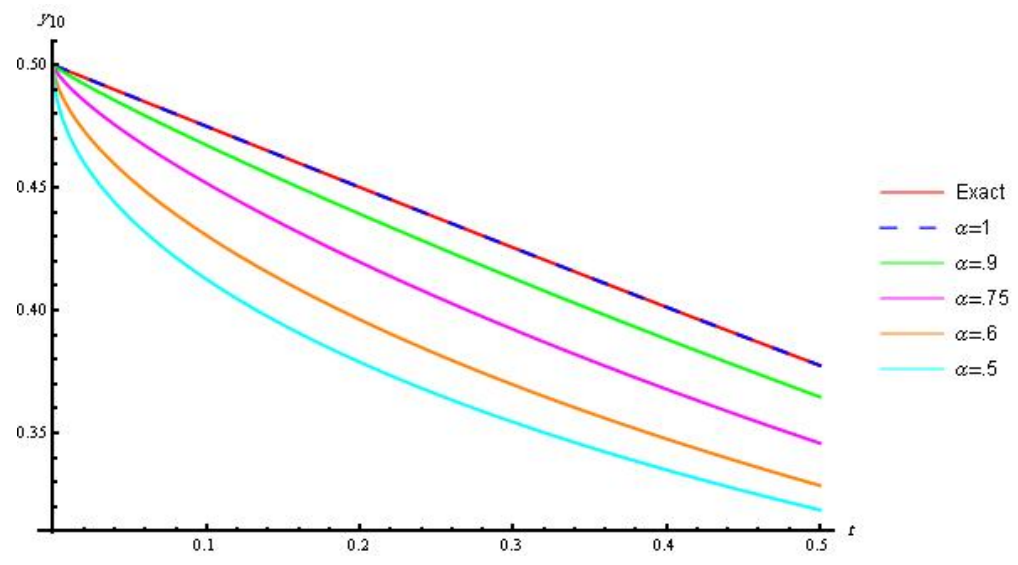

Figure 3

\section{Conclusions}

In this paper we used the residual power series (RPS) method to study the solution of the fractional Riccati equation. We reached to the fact that RPS is very practical and efficient in finding analytical solutions and in addition numerical solutions for wide classes of linear and nonlinear fractional differential equations. It provides more series solutions that converge very rapidly in real physical problems. Three concrete examples were tested to manifest the validity of the proposed method and the obtained results were remarkable and compatible with other methods. Moreover, we noticed that the obtained approximate solutions for various values of $0<\alpha \leqslant 1$ continuously communicate until the first order derivative is reached. This is another indicator that our results are likely to be legitimate.

\section{References}

[1] O. Abu Arqub, Series solution of fuzzy differential equations under strongly generalized differentiability, J. Adv. Res. Appl. Math., 5 (2013), 31-52. 1, 2, 3

[2] O. Abu Arqub, A. El-Ajou, Z. Al Zhour, S. Momani, Multiple solutions of nonlinear boundary value problems of fractional order: a new analytic iterative technique, Entropy, 16 (2014), 471-493. 1, 2

[3] M. Alquran, Analytical solution of time-fractional two-component evolutionary system of order 2 by residual power series method, J. Appl. Anal. Comput., 5 (2015), 589-599.

[4] M. Alquran, K. Al-Khaled, J. Chattopadhyay, Analytical solutions of fractional population diffusion model: residual power series, Nonlinear Stud., 22 (2015), 31-39.

[5] M. Alquran, K. Al-Khaled, T. Sarda, J. Chattopadhyay, Revisited Fisher's equation in a new outlook: a fractional derivative approach, Phys. A, 438 (2015), 81-93. 1

[6] B. D. O. Anderson, J. B. Moore, Optimal control: linear quadratic methods, Prentice-Hall, New Jersey, (1990). 1

[7] M. Caputo, Linear models of dissipation whose Q is almost frequency independent, II, Reprinted from Geophys. J. R. Astr. Soc., 13 (1967), 529-539, Fract. Calc. Appl. Anal., 11 (2008), 4-14. 2

[8] M. Caputo, F. Mainardi, A new dissipation model based on memory mechanism, Reprinted from Pure Appl. Geophys., 91 (1971), 134-147, With an editorial note by Virginia Kiryakova, Fract. Calc. Appl. Anal., 10 (2007), 309-324. 2

[9] A. El-Ajou, O. Abu Arqub, Z. Al Zhour, S. Momani, New results on fractional power series: theories and applications, Entropy, 15 (2013), 5305-5323. 1, 2, 3 
[10] A. El-Ajou, O. Abu Arqub, S. Momani, Approximate analytical solution of the nonlinear fractional KdV-Burgers equation: a new iterative algorithm, J. Comput. Phys., 293 (2014), 81-95. 1

[11] A. El-Ajou, O. Abu Arqub, S. Momani, D. Baleanu, A. Alsaedi, A novel expansion iterative method for solving linear partial differential equations of fractional order, Appl. Math. Comput., 257 (2015), 119-133. 1

[12] J. F. Gómez-Aguilar, R. Razo-Hernández, D. Granados-Lieberman, A physical interpretation of fractional calculus in observables terms: analysis of the fractional time constant and the transitory response, Rev. Mexicana Fís., 60 (2014), 32-38. 2

[13] M. Inc, Z. S. Korpinar, M. M. Al Qurashi, D. Baleanu, A new method for approximate solutions of some nonlinear equations: Residual power series method, Adv. Mech. Eng., 8 (2016), 1-7. 1

[14] H. Jafari, H. Tajadodi, He's variational iteration method for solving fractional Riccati differential equation, Int. J. Differ. Equ., 2010 (2010), 8 pages. 1

[15] N. A. Khan, A. Ara, N. A. Khan, Fractional-order Riccati differential equation: analytical approximation and numerical results, Adv. Difference Equ., 2013 (2013), 16 pages. 1, 4, 4

[16] S. Kumar, A. Kumar, D. Baleanu, Two analytical methods for time-fractional nonlinear coupled Boussinesq-Burger's equations arise in propagation of shallow water waves, Nonlinear Dynam., 85 (2016), 699-715. 1

[17] A. Kumar, S. Kumar, M. Singh, Residual power series method for fractional Sharma-Tasso-Olever equation, Commun. Numer. Anal., 2016 (2016), 1-10. 1

[18] Y.-L. Li, Solving a nonlinear fractional differential equation using Chebyshev wavelets, Commun. Nonlinear Sci. Numer. Simul., 15 (2010), 2284-2292. 1, 4

[19] A. Neamaty, B. Agheli, R. Darzi, The shifted Jacobi polynomial integral operational matrix for solving Riccati differential equation of fractional order, Appl. Appl. Math., 10 (2015), 878-892. 1

[20] Z. Odibat, S. Momani, Modified homotopy perturbation method: application to quadratic Riccati differential equation of fractional order, Chaos Solitons Fractals, 36 (2008), 167-174. 1, 4, 4

[21] I. Podlubny, Geometric and physical interpretation of fractional integration and fractional differentiation, Dedicated to the 60th anniversary of Prof. Francesco Mainardi, Fract. Calc. Appl. Anal., 5 (2002), 367-386. 2

[22] N. H. Sweilam1, M. M. Khader, A. M. S. Mahdy, Numerical studies for solving fractional Riccati differential equation, Appl. Appl. Math., 7 (2012), 595-608. 1

[23] F. Xu, Y.-X. Gao, X. Yang, H. Zhang, Construction of fractional power series solutions to fractional Boussinesq equations using residual power series method, Math. Probl. Eng., 2016 (2016), 15 pages. 1

[24] J.-J. Yao, A. Kumar, S. Kumar, A fractional model to describe the Brownian motion of particles and its analytical solution, Adv. Mech. Eng., 7 (2015), 1-11. 1 